\title{
Therapeutic MUC1-Based Cancer Vaccine Expressed in Flagella_Efficacy in an Aggressive Model of Breast Cancer
}

\author{
Nathalie Machluf, Ruth Arnon* \\ Department of Immunology, The Weizmann Institute of Science, Rehovot, Israel. \\ Email: *ruth.arnon@weizmann.ac.il \\ Received March $5^{\text {th }}, 2012$; revised April $7^{\text {th }}, 2012$; accepted May $8^{\text {th }}, 2012$
}

\begin{abstract}
MUC1, a tumor-associated antigen overexpressed in many carcinomas, represents a candidate of choice for cancer immunotherapy. Flagella-based MUC1 vaccines were tested in therapeutic setting in two aggressive breast cancer models, comprising the implantation of the 4T1-MUC1 cell line in either Balb/c, or Human MUC1 transgenic mice in which spontaneous metastases occur. Recombinant flagella carrying only 7 amino acid of MUC1 elicited therapeutic activity, affecting both the growth of established growing tumors and the number of metastases. Higher therapeutic activity was achieved with an additional recombinant flagella designed with the SYFPEITHI algorithm. The vaccines triggered a Th1 response against MUC1 with no evident autoimmune response towards healthy MUC1-expressing tissues. Recombinant flagella carrying a 25-residue fragment of MUC1, induced the most effective response, as evidenced by a significant reduction of both the size and growth rate of the tumor as well as by the lower number of metastases, and expanding life span of vaccinated mice.
\end{abstract}

Keywords: Breast Cancer; Flagella; Vaccine; Immunization; Prophylactic; MUC1; Transgenic Mice; Tumor Growth

\section{Introduction}

In spite of pronounced improvements in cancer management over the last decade, traditional cancer treatments remain limited in their therapeutic capacity and are accompanied by adverse side effects. There is therefore a pressing demand for new therapeutic approaches. Cancer vaccines, based on tumor associated-antigens, represent an attractive therapeutic strategy. They are aimed at inducing a specific immune response towards the tumor, they are usually not associated with toxic side effects and they can establish a long-term immune memory, which is critical in preventing tumor recurrence [1].

The tumor associated antigen mucin 1 (MUC1) is a high molecular weight transmembrane glycoprotein expressed on the apical surface of most of the glandular epithelial cells [2]. Its large extracellular domain contains 25-125 tandem repeats (depending on the allele) of the 20 amino acids sequence GVTSAPDTRPAPGSTAP$\mathrm{PAH}$, which includes a well known $\mathrm{B}$ and $\mathrm{T}$ cell immunodominant epitopes in mouse and in human (APDTRPA) [3-6]. In malignant cells, MUC1 is over-expressed, redistributed over the full surface of the cells, and hypoglycosylated [3]. Consequently, its antigenicity is different

\footnotetext{
*Corresponding author.
}

in malignant and normal cells, which allows the immune system to distinguish between them [1]. Moreover, MUC1 expression has been directly correlated with the aggressiveness of the tumors and poor survival of the patients. Thus, MUC1 appears to be a suitable target for treatment even in advanced disease stage [4]. During recent years, the utilization of MUC1 in immunotherapeutic approaches for the development of either peptide, carbohydrate, DNA or dendritic cells-based vaccines, was reported. Indeed, promising results with such vaccines were achieved in animal models, but moving to the clinical arena, several trials using MUC1 based vaccines, employing different carriers and/or adjuvant, did not exhibit sufficient efficacy $[5,6]$.

The carrier system employed in this study has been designed to stimulate the immune response to linear epitopes by their expression in flagellin, the structural subunit of the flagellum filament [7]. In previous studies carried out in our laboratory, it was demonstrated that immunization with recombinant flagella expressing epitopes of various viral and bacterial pathogens evoked humoral as well as cellular immune responses against the inserted epitope, which resulted in protection against a challenge infection [8-14]. In these studies, the flagella, which is a known ligand of the Toll-like receptor 5 [15], 
acted as both carrier and adjuvant thus rendering superfluous the need for external adjuvant. Furthermore, the flagella did not induce a carrier suppression effect on the immune response against the inserted epitope [16]. However, notwithstanding its strong immunostimulatory properties, this recombinant vaccine approach has not been investigated hitherto for its possible effect on tumor growth.

In the present study, we explored the potential use of several epitope-based recombinant flagella vaccines targeting the extracellular TR sequence of MUC1, selected using the SYFPEITHI database [17]. We mainly focused on the therapeutic application of the vaccines and demonstrated a significant decrease of the growth of established tumors in two animal models. As shown in the following, the flagellin MUC1-based vaccines induced a Th1 type response against MUC1, and were not associated with autoimmune symptoms. Furthermore, in the mouse model equivalent to grade IV breast cancer, these chimeric vaccines limited significantly the metastatic process.

\section{Materials and Methods}

\subsection{Cell Line}

The 4T1-MUC1 cell line was kindly provided by Prof. T.M. Allen (University of Alberta, Edmonton, Canada) [18]. Cells were cultured in DMEM supplemented with $10 \%$ foetal bovine serum from Gibco BRL (both from Life Technologies), $60 \mu \mathrm{m}$ thioguanine from Sigma, 1 mM MEM non-essential amino acids, penicillin (100 units $/ \mathrm{mL})$, streptomycin $(100 \mu \mathrm{g} / \mathrm{mL})$, and geneticin (400 $\mu \mathrm{g} / \mathrm{mL}$ ), all supplied by Biological industries. The frequent checking of MUC1 expression was performed by FACS with anti-MUC1 antibody from Serotec.

\subsection{Mice}

Female Balb/c mice ( 8 weeks old) were purchased from Harlan Laboratories (Jerusalem). Human MUC1 transgenic mice $(\mathrm{C} 57 \mathrm{BL} / 6)$ were kindly provided by Prof. Gendler S. (Mayo Clinic, Scottsdale, AZ). These transgenic mice express the human MUC1 in the same pattern as humans do and are tolerant to the human MUC1 [19]. Human transgenic mice were crossed with $\mathrm{Balb} / \mathrm{c}$ mice and experiments were carried out either with the first generation of offspring (F1) or with the eighth generation (F8) considered cleared from the C57BL/6 background. Animals were handled according to the Institutional Animal Care and Use Committee of the Weizmann Institute.

\subsection{PCR Screening of MUC1 Positive Transgenic Mice}

PCR was used to routinely identify MUC1 transgenic positive mice during the successive crossing. PCR was carried out in a total volume of $50 \mu \mathrm{L}$ with the following reagents: $0.65 \mu \mathrm{M}$

5'-CTTGCCAGCCATAGCACCAAG-3' (bp 745 - 765) and $0.65 \mu \mathrm{M} 5$ '-CTCCACGTCGTGGA CATTGATG-3' (bp 1086 - 1065) primer, $10 \mu \mathrm{L}$ of ready mix (Larova), 1 $\mu \mathrm{L}$ of tail DNA and DDW. The amplification program consisted of one cycle of $10 \mathrm{~min}$ at $94^{\circ} \mathrm{C}$ and 40 cycles of 1 min each at 94,61 and $72^{\circ} \mathrm{C}$. The PCR product of each reaction was analysed by size fractionation through a $1 \%$ agarose gel. Amplification of MUC1 positive DNA resulted in a 500 bp fragment.

\subsection{Preparation of Recombinant Flagella}

The following oligonucleotides were synthesized:

the oligonucleotide 5'-GCT CCG GAT ACC CGT CCG GCT GAT-3' coding for the 7 amino acids epitope APDTRPA (MUC1.7), the oligonucleotide 5'-AGA CCG GCT CCG GGT AGC ACC GCT CCG GAT-3' coding for the 9 amino acids epitope RPAPGSTAP (MUC1.9) identified by the SYFPEITHI database, and the oligonucleotide sequence of 25 amino acids

GVTSAPDTRPAPGSTAPPAHGVTSA (MUC1.25)

5'-GGCGTGACCTCGGCGCCGGAT

ACCCGCCCGGCGCCGGGCTCGACCGCGCCGCC GGCGCATGGCGTGACCTCGGCG-3'. For the two first oligonucleotides the last 3 nucleic acids GAT were added in order to preserve the EcoRV restriction site in the flagellin sequence. Codon usage was according to the sequence of the flagellin gene.

The plasmid vector carrying the flagellin gene from Salmonella munchen pls408 (Newton S. M., Jacob C. O., Stocker B. A., 1989, Science. 244: 70-2) was used for the expression of the epitopes MUC1.7 and MUC1.9 at the EcoRV restriction site. For the insertion of MUC1.25, the plasmid pls408 was slightly modified by mutagenesis (Stratagene) to create an Age I restriction site 21 bp in 5' of the EcoRV site. The recombinant plasmids were transformed into E. coli JM101 competent cells by heat chock. Plasmids from positive colonies were purified and used to transform a flagellin negative live vaccine strain (an Aro A mutant) of S. Dublin SL5928 by electroporation. The transformed S. Dublin cells were selected for Ampicillin resistance, motility under the light microscope and growth in semisolid agar plates. The flagella comprising the hybrid flagellins were detached from the bacteria using acidic cleavage as described elsewhere (Ibrahim $e t a l$.). The purity of the isolated peptides was assessed by SDS-PAGE.

The recombinant flagellin harbouring MUC1.7 was denoted Fla-MUC1.7, the flagellin carrying MUC1.9 was denoted Fla-MUC1.9, the flagellin carrying MUC1.25 was denoted Fla-MUC1.25 and a flagellin bearing a non relevant peptide (TYQRTRALVRTG) was denoted FlaNRP and serves as a control vaccine. 


\subsection{Immunization Procedure in Vaccination Experiments}

In the prophylactic immunization experiment, female Balb/c mice ( 8 weeks old) were immunized subcutaneously (s.c.) 3 times at 4 weeks intervals with Fla-MUC1.7 (100 $\mu \mathrm{g} /$ mouse), Fla-NRP (100 $\mu \mathrm{g} /$ mouse) or PBS in adjuvant (complete Freund's adjuvant for the first immunization and incomplete Freund's adjuvant for the boosts, both supplied by Sigma). Four months after the last immunization, 1.5 million 4T1-MUC1 tumor cells were subcutaneously implanted in a total volume of $100 \mu \mathrm{L}$.

In therapeutic vaccination experiments, mice were implanted subcutaneously (s.c.) with 1.5 million 4T1-MUC1 cells in a total volume of $100 \mu \mathrm{l}$ in PBS. Around two weeks post-implantation, the average tumor size was around $0.1 \mathrm{~cm}^{3}$ in each group, and immunization was performed such as $100 \mu \mathrm{g}$ of Fla-MUC1.7 or $100 \mu \mathrm{g}$ of Fla-MUC1.9 or $50 \mu \mathrm{g}$ of each of them (when used in combination), or $100 \mu \mathrm{g}$ of Fla-MUC1.25, or Fla-NRP, or PBS, were administrated to the mice bearing tumor. The dose of $100 \mu \mathrm{g}$ was selected as optimal after a dose range finding experiment using doses ranging from 20 to $200 \mu \mathrm{g}$. Fifty micrograms was identified as the minimal therapeutic effective dose, whereas $200 \mu \mathrm{g}$ seemed less tolerated by the mice.

The average tumor growth was calculated as the difference between the tumor size on a given day and the tumor size on the first day of immunization. Variability in tumor growth inter experiments was observed. The end of each experiment was dictated by the time when mice became moribund.

\subsection{Tumor Size Determination and Tumor Growth Calculation}

The area on the back of each mouse, where tumor cells were implanted as described in Section 2.5 was first inspected visually to localise the formed mass. The initial detection of the tumor mass was either confirmed or further performed by palpation. Furs on and around the tumor were cut as short as possible in order to facilitate and maximize the accuracy of the tumor measure. The tumor volume was determined using the equation: volume $=0.4$ $a b^{2}$, where $a$ and $b$ are respectively the larger and the smaller diameter of the tumor, measured using a calliper.

\subsection{Antibody Isotyping}

Tumor bearing mice were bled from the heart around 20 days post-immunization. Mice "non bearing tumor" were immunized s.c. 3 times 4 weeks intervals with $100 \mu \mathrm{g}$ of Fla-MUC1.7, or $100 \mu \mathrm{g}$ of Fla-MUC1.9, or $50 \mu \mathrm{g}$ of Fla-MUC1.7 + $50 \mu \mathrm{g}$ of Fla-MUC1.9, or 100 $\mu \mathrm{g}$ of Fla-NRP, or PBS, with adjuvant (first immunization in CFA and further boost in IFA). Bleeding from the eye were performed 2 weeks after each immunization. ELISA was performed using Nunc Maxisorp plate coated overnight at $4^{\circ} \mathrm{C}$ with $\mathrm{BSA}$ coupled to the 20 amino acids sequence of the tandem repeat of MUC1 GVTSAPDTRPAPGSTAPPAH $(10 \mu \mathrm{g} /$ well in $100 \mu \mathrm{L})$. The plate was washed twice with PBS containing $0.1 \%$ Tween-20 (PBS-Tween). The wells were then blocked with $1 \%$ BSA in PBS for $1 \mathrm{~h}$ at $37^{\circ} \mathrm{C}$, and washed with PBS-Tween. The serum obtained from mice of each group was pooled and $50 \mu \mathrm{L}$ samples were added in duplicate. The plates were incubated for 2 hours at $37^{\circ} \mathrm{C}$, and washed in PBS-Tween. Goat anti-mouse IgG2a, IgG3 (conjugated to horseradish peroxidase-HRP) or IgG1 (conjugated to alkaline phosphase-AP) were used as second antibodies (Jackson Laboratories). 3.3',5,5'tetramethylbezidine (TMB, Sigma) and alkaline phosphatase substrate solution (Sigma) were added as substrate. Following the addition of the substrate $(50 \mu \mathrm{L} /$ well), the reaction was allowed to proceed, and was terminated by adding $50 \mu \mathrm{L}$ of 1HCL (for HRP) or $15 \mu \mathrm{L}$ of $3 \mathrm{~N} \mathrm{NaOH}$ (for AP). The intensity of colour was subsequently determined at $450 \mathrm{~nm}$ and $405 \mathrm{~nm}$, respecttively by an ELISA reader (Multiscan MCC/340 MK II, Lab system).

\subsection{INF $y$ and IL-4 Secretion Assay}

Female Balb/c mice ( 8 weeks old) were s.c. immunized 4 times 4 weeks intervals with $50 \mu \mathrm{g}$ per mouse of each of Fla-MUC1.7 and Fla-MUC1.9, or Fla-NRP, or PBS with adjuvant (first immunization with complete Freund's adjuvant, and further boosts in incomplete Freund's adjuvant both from Sigma). Ten days after the last immunization, spleens were removed and the INF $\gamma$ secretion assay and IL-4 secretion assay using Miltenyl Biotec kit. Splenocytes were stimulated for 16 hours at $37^{\circ} \mathrm{C}$ with 5 $\times 10^{5}$ killed $4 \mathrm{~T} 1-\mathrm{MUC} 1$ cells (by several freeze thaw cycles) in RPMI (Gibcobrl, Life Technologies) supplemented with $5 \%$ fresh Balb/c mouse serum. The rest of the procedure was performed according to the manufacturer's instructions.

\subsection{Histopathological Analysis}

The histopathological analysis was performed on few of the organs expressing MUC1: the lung, the liver and the kidneys. MUC1 transgenic mice bearing tumor were immunized with $50 \mu \mathrm{g}$ of Fla-MUC1.7 $+50 \mu \mathrm{g}$ of FlaMUC1.9 or PBS, 10 days post-immunization and the organs of interest were taken out 21 days post-immunization. The naive MUC1 transgenic animals (i.e. non-bearing tumor) were immunized 4 times 4 weeks intervals with the same preparation as in tumor-bearing mice, with adjuvant (first immunization with CFA and further boosts in IFA). The same organs of interest were removed 4 weeks 
after the last immunization. A phosphate buffered $4 \%$ paraformaldehyde fixative were used overnight RT. Histological sections were prepared and staining with hematoxylin and eosin were made by the histology unit of Weizmann Institute. The presence of any symptoms of autoimmunity, such as architectural damage or cellular infiltrate was searched under light microscopy.

\subsection{Statistical Analysis}

Normality was tested using the test of Shapiro-Wilk on each group at each time point. The program SAS was used to perform a two way (or factorial) ANOVA (factors being time and treatment) with repeated measure on one factor (time) finding that an interaction one way ANOVA twice. Once comparing the treatment per each time point in which case subject was treated as blocks, and once comparing time per each treatment. Significant results were followed by Fisher's LSD multiple comparison.

\section{Results}

\subsection{Immunization with a Single Epitope Recombinant Vaccine Fla-MUC1.7}

\subsubsection{Therapeutic Vaccination with Fla-MUC1.7 in Balb/c Mice}

In order to validate the potential use of recombinant flagella as a MUC1-based cancer vaccine, we initially tested the therapeutic capacity of a recombinant vaccine denoted Fla-MUC1.7 carrying the 7 residues immunodominant epitope (APDTRPA) [3-6]. The administration of Fla-MUC1.7 was carried out in groups of mice bearing each a tumor of average size $0.1 \mathrm{~cm}^{3}$, formed 15 days post-implantation of 4T1-MUC1 cells. Tumor growth was calculated by the difference between the tumor size on a given day and the tumor size on the day of immunization (which defines the day 0 of the experiment). Twelve days post-immunization (which is equivalent to 27 days post-implantation of $1.5 \times 10^{6} 4 \mathrm{~T} 1$ MUC1 cells), the tumor growth was 3-fold lower as a result of the single administration of Fla-MUC1.7 compared to the growth monitored in control mice immunized either with a control recombinant vaccine denoted Fla-NRP (for non relevant peptide) or with PBS $(p<0.05)$ (Figure 1(a)). It was also demonstrated that the therapeutic activity of Fla-MUC1.7 didn't required the presence of an adjuvant (complete Freund's adjuvant) (data not shown).

\subsubsection{Therapeutic Vaccination with Fla-MUC1.7 in MUC1-Transgenic Mice}

To further assess the efficacy of Fla-MUC1.7 in reducing tumor growth, another breast cancer animal model was used, consisting of implanting the same tumor cells (4T1-MUC1) in offspring obtained upon crossing human MUC1 transgenic mice and Balb/c mice. MUC1 transgenic mice $(\mathrm{F} 1)$, bearing each a $4 \mathrm{~T} 1-\mathrm{MUC1}$ tumor of average size $0.1 \mathrm{~cm}^{3}$ (10 days after cells implantation),
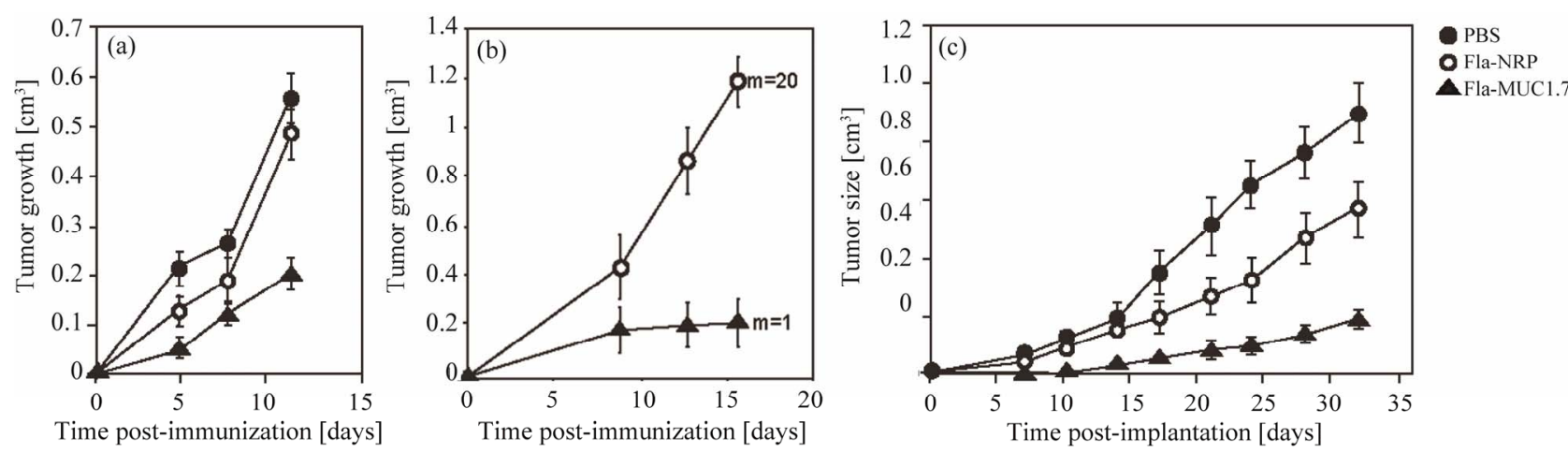

Figure 1. Reduction of tumor growth by recombinant flagella carrying a single epitope of MUC1. Full circle: PBS; Empty circle: Fla-NRP; Triangle: Fla-MUC1.7; (a-b) Therapeutic vaccination: recombinant flagella was administered to mice bearing growing tumors. (a) On the day of the immunization (day 0), group of Balb/c mice bearing 4T1-MUC1 tumor of $0.1 \mathrm{~cm}^{3}$ on the average were immunized with the different preparations as indicated. The average tumor growth (which is the difference between tumor size on a given day and the tumor size on the first day of immunization (day 0$) \pm$ s.e. of each group (n = 10 to 15) is presented. The reduction of tumor growth observed in mice immunized with Fla-MUC1.7 as compared to control groups (p < 0.01); (b) On the day of the immunization (day 0), group of human MUC1 transgenic mice bearing 4T1-MUC1 tumor of $0.1 \mathrm{~cm}^{3}$ in average were immunized with the different preparations as indicated. The average tumor growth (which is the difference between tumor size on a given day and the tumor size on the first day of immunization day 0 ) \pm s.e. of each group $(n=5)$ is presented. A significant reduction of tumor growth was observed in mice immunized with Fla-MUC1.7 as compared to control groups $(p<\mathbf{0 . 0 1})$. The number of lung metastases counted in one representative mouse per group is indicated (m); (c) Prophylactic vaccination: naive Balb/c mice were pre-immunized 3 times at 3 weeks intervals four months prior 4T1-MUC1 cells implantation. The average tumor size \pm s.e. of each group $(n=8$ to 10) is presented. Tumor appearance was delayed and growth was significantly slower in mice pre-immunized with Fla-MUC1.7 than in control animals $(\mathrm{p}<0.01)$. 
were immunized either with Fla-MUC1.7 or with FlaNRP. Sixteen days later, the tumor growth was 6 times smaller in immunized mice as compared to animal administered with the control Fla-NRP $(\mathrm{p}<0.01)$ (Figure 1(b)). In this model, metastases were detected and counted in the lungs in one representative animal of the average tumor growth of each group. Only one lung metastase was detected in the mouse immunized with Fla-MUC1.7, whereas 20 lung metastases were counted in the mouse administered with the control vaccine Fla-NRP. These results are indicative of the efficacy of Fla-MUC1.7 in reducing not only tumor growth, but also the metastasic process.

\subsubsection{Prophylactic Vaccination with Fla-MUC1.7 in Balb/c Mice}

In contrast to vaccines for infectious diseases, which are used in prophylaxis, the major application of cancer vaccines would be therapeutic (i.e. after cancer diagnosis). However, prophylactic cancer vaccination could satisfy the unmet medical need of at-risk subjects (e.g. carriers of mutation on BRCA1 or BRCA2) for whom frequent surveillance, preventive chemotherapy or total mastectomy are the only alternatives at present. It was therefore of interest to explore the prophylactic activity of FlaMUC1.7.

Balb/c mice were injected 3 times with Fla-MUC1.7, Fla-NRP, or PBS, and were challenged with 4T1-MUC1 cells 4 months after the last immunization, in order to assess whether the vaccine could ensure a long-term protection. Tumor appearance was delayed to 14 days after implantation in mice immunized with Fla-MUC1.7, whereas in the control groups (PBS or Fla-NRP) the tumors were detected already after 7 days (Figure 1(c)). Furthermore, 32 days post-implantation, the tumor size in the Fla-NRP control group was about $40 \%$ smaller than in the PBS control group, possibly due to the adjuvant effect of the flagella. However, at this time point, the average tumor size in Fla-MUC1.7 immunized mice was 5 to 6 fold smaller than in the PBS control group ( $p<$ $0.01)$. These results indicate that Fla-MUC1.7 is adequate also for prophylactic vaccination.

\subsection{Immunization Combining Two Recombinant Vaccines Fla-MUC1.7 and Fla-MUC1.9}

The rationale behind the design of Fla-MUC1.7, as a vaccine for MUC1 expressing cancers, relies on the recruitment of a certain immune repertoire against the 7 amino acid of the TR of MUC1 expressed on tumor cells. Therefore, we examined whether immunization with FlaMUC1.7 together with another recombinant Flagella MUC1-based preparation, aimed at recruiting a different immune repertoire, would have an additive therapeutic effect.

\subsubsection{Choice of another MUC1 Epitope by Using SYFPEITHI Database}

Using the MHC binding prediction software SYFPEITHI [16], epitope(s) within the tandem repeat of MUC1 for high affinity with $\mathrm{K}^{\mathrm{d}}$ and $\mathrm{L}^{\mathrm{d}}$ was searched. The 9 amino acids peptide RPAPGSTAP was identified as displaying the highest score $($ score $=16)$. It is of interest to note that the epitope APDTRPA previously used in Fla-MUC1.7 have a smaller score (score $=11$ ). Therefore, recombinant flagella expressing RPAPGSTAP, denoted Fla-MUC1.9, was prepared and evaluated for its efficacy in combination with Fla-MUC1.7.

\subsubsection{Therapeutic Vaccination with Fla-MUC1.7 and Fla-MUC1.9 in Balb/c Mice}

Mice bearing a 4T1-MUC1 tumor of average size 0.1 $\mathrm{cm}^{3}$ were immunized with either one of the vaccines (Fla-MUC1.7 or Fla-MUC1.9) or with the combined preparations. Mice immunized with Fla-MUC1.7 or FlaMUC1.9 alone display a similar tumor growth, which were approximately 2 fold smaller than in the PBS control group ( $p<0.01)$ (Figure $2(\mathbf{a})$ ). The tumor growth in mice administered with the combination of the two vaccines Fla-MUC1.7 and Fla-MUC1.9 was significantly lower than the tumor growth assessed in mice that received only one of these preparations $(p<0.05)$. These results demonstrate the additive effect of the 2 vaccines administered together.

\subsubsection{Multiple Administration of Fla-MUC1.7 and Fla-MUC1.9}

The therapeutic efficacy upon the injection of FlaMUC1.7 alone or in combination with Fla-MUC1.9 described in Figure 2(b) was performed after a single injection in mice bearing tumor of $0.1 \mathrm{~cm}^{3}$ in average. We further investigated the benefit of a multiple immunizations schedule consisting of 2 additional administrations at one week interval. As shown in Figure 2(b), the two subsequent injections of Fla-MUC.1.7 plus Fla-MUC1.9 did not have a significant effect on the tumor growth. This finding was further supported by data collected in two groups of MUC1 transgenic mice bearing tumor, immunized either once or three times (at weekly intervals), displaying identical tumor growth (data not shown). However, the remarkable anti-tumor effect using the combined vaccines showed in Figure 2(b) is to be noticed. Upon the first administration tumor size even decreased in mice immunized with Fla-MUC1.7 plus Fla-MUC1.9, and after 18 days the tumor growth was 7 fold smaller in mice immunized with the combined vaccines than that of mice injected with PBS $(p<0.01)$. At this same day, mice from the control groups (PBS and Fla-NRP) had to be euthanized for ethical reasons, whereas the tumor size in mice immunized with Fla- 

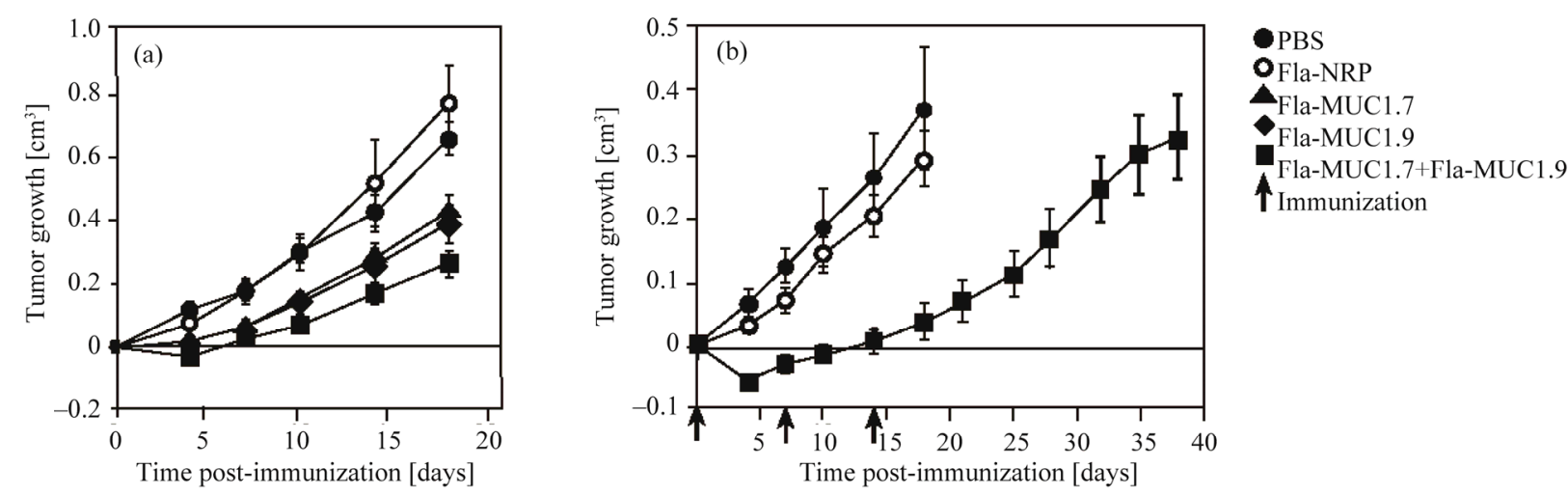

Figure 2. Additive therapeutic activity of Fla-MUC1.7 and Fla-MUC1.9 in Balb/c mice bearing tumors. Full circle: PBS; Empty circle: Fla-NRP; Triangle: Fla-MUC1.7; Diamond: Fla-MUC1.9; Square: Fla-MUC1.7 + Fla-MUC1.9; Arrow: Immunizaion. On the day of the immunization (day 0), group of Balb/c mice bearing 4T1-MUC1 tumor of $0.1 \mathrm{~cm}^{3}$ in average were immunized with the different preparations. The average tumor growth (which is the difference between tumor size on a given day and the tumor size on the first day of immunization) \pm s.e. of each group $(n=10$ to 15) is presented; (a) A significant reduction of tumor growth was observed in mice immunized with Fla-MUC1.7 + Fla-MUC1.9 as compared to mice immunized with only one of the vaccine $(p<0.05)$, in which the tumor growth was already significantly slower than in control groups ( $<$ 0.01); (b) The reduction of tumor growth observed in Balb/c mice immunized with Fla-MUC1.7 + Fla-MUC1.9 as compared to control groups $(p<0.01)$, upon the first immunization. Subsequent immunization, indicated by arrows, didn't further impact the tumor growth.

MUC1.7 plus Fla-MUC1.9 was similar to tumor size on the first day of immunization, and its rate of growth was rather slow. When immunizations were discontinued and tumor growth was accelerated, 38 days after the first immunization, or 24 days after the last vaccination, the mice immunized with Fla-MUC1.7 and Fla-MUC1.9 had to be euthanized too. But, as shown, the life span of mice vaccinated with Fla-MUC1.7 and Fla-MUC1.9 was more than doubled as compared to control mice.

\subsection{Immune Response Triggered by the Recombinant Flagellin MUC1-Based Vaccines}

The flagellin is a ligand associated with the Toll like receptor 5, which belongs to the Toll-like receptors family well documented to favour a Th1 response [19], which may contribute to its activity as an adjuvant [20-23].

\subsubsection{Splenocytes from Balb/C Mice Immunized with Fla-MUC1.7 and Fla-MUC1.9 Secrete INF upon Stimulation by 4T1-MUC1 Killed Cells}

To elucidate the immune mechanism triggered by the administration of Fla-MUC1.7 and Fla-MUC1.9, we assessed the percentage of splenocytes collected from $\mathrm{Balb} / \mathrm{c}$ mice immunized either with the combined vaccines, or with a control preparation (Fla-NRP) or with PBS, secreting INFy or IL-4 in response to in vitro stimulation with 4T1-MUC1 pre-killed cells. As depicted in Figure 3, a high proportion of splenocytes secreting INF $\gamma$ $(1.3 \%)$ was demonstrated only in mice immunized
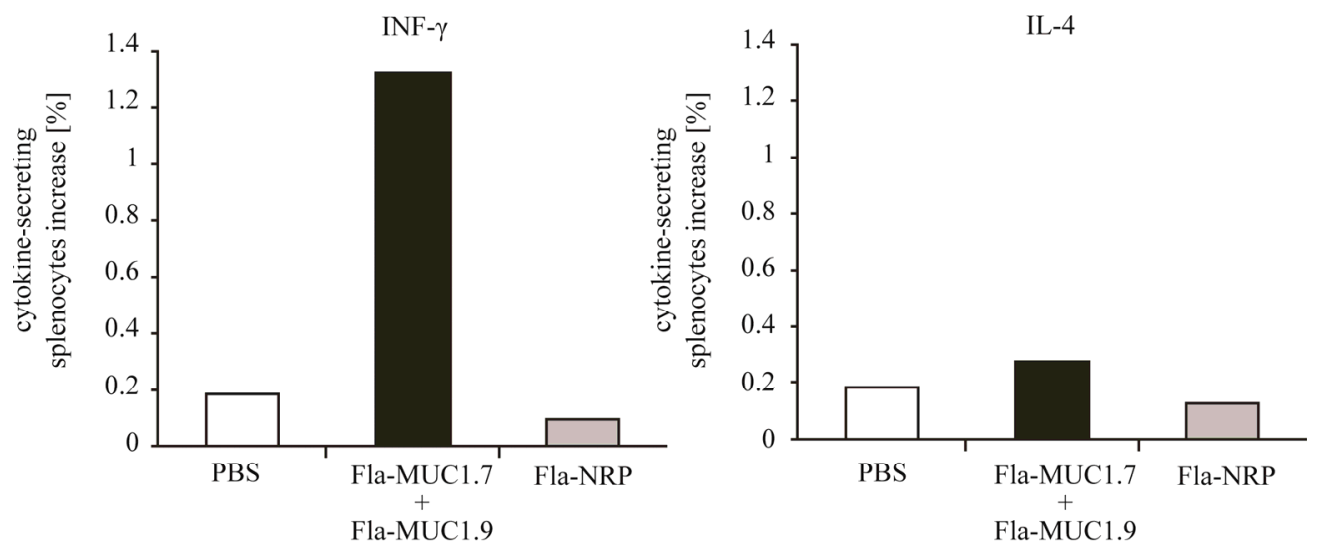

Figure 3. Increase of INF $\gamma$ secretion by splenocytes isolated from Balb/c mice immunized with Fla-MUC1.7 and Fla-MUC1.9. The results indicate the difference between the percentage of cells secreting the cytokine of interest after exposure to killed tumor cells and to medium alone; Results were obtained from samples in which 3 spleens for each group were pooled. 
with Fla-MUC1.7 and Fla-MUC1.9. We could also notice a slight higher proportion of splenocytes secreting IL-4 in mice immunized with the combined preparation $(0.3 \%)$ as compared to the PBS control group $(0.18 \%)$, but the significance of this difference is unclear. These data demonstrate the induction of Th1 type response towards MUC1 upon vaccination with Fla-MUC1.7 and Fla-MUC1.9.

\subsubsection{Antibody Isotypes}

Another aspect of the type of immune response generated by Flagellin MUC1-based cancer vaccines is reflected in the antibody isotypes profile anti-MUC1 in the serum of MUC1 transgenic mice bearing tumors that were either immunized with Fla-MUC1.7 and Fla-MUC1.9 or administered with PBS. In order to characterize the spontaneous immune response of these transgenic mice to 4T1-MUC1 tumor cells, the level of antibodies was compared to that of naive mice that were not exposed to the tumor cells. The results displayed in Figure 4 show that only IgG1 anti-MUC1 titer was significantly higher in mice bearing tumor administered with PBS as compared to naive mice $(\mathrm{p}<0.05)$, and the response in the Fla-MUC1.7 plus Fla-MUC1.9 immunized mice was even higher $(\mathrm{p}<0.01)$. These results suggested that $4 \mathrm{~T} 1-$ MUC1 tumor cells naturally triggered a type 2 response

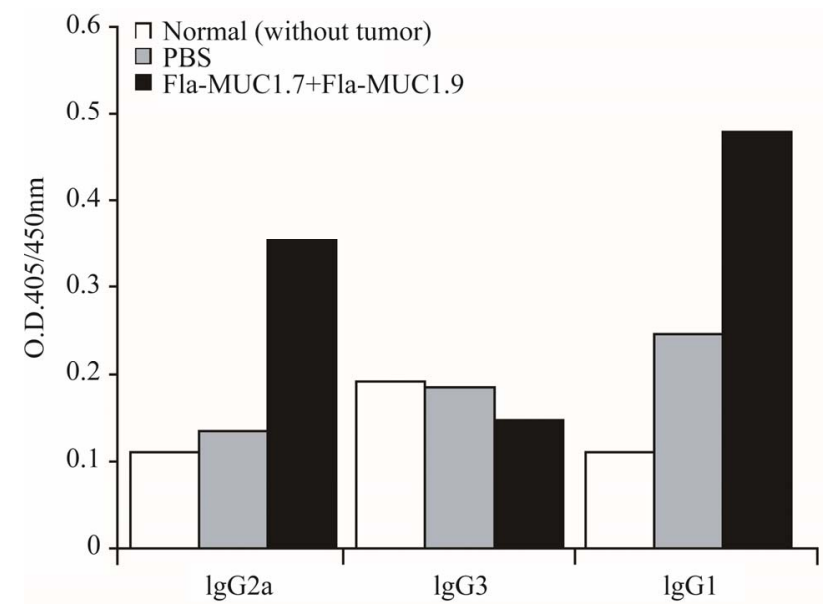

Figure 4. Antibody isotype secretion in MUC1 transgenic mice bearing tumors, immunized with Fla-MUC1.7 and Fla-MUC1.9. White bar: Naïve mice; Grey bar: PBS; Black bar: Fla-MUC1.7 + Fla-MUC1.9. IgG1, IgG2a and IgG3 anti-MUC1 antibodies titre in serum isolated from MUC1 transgenic mice bearing tumor 32 days post-immunized with the different preparations as indicated in the legend. IgG1 anti-MUC1 titer was significantly higher in mice bearing tumor immunized with Fla-MUC1.7 plus Fla-MUC1.9 $(p<0.01)$, or in mice administered with PBS $(p<0.05)$ as compared to naive mice. IgG2a was detected only in mice immunized with Fla-MUC1.7 plus Fla-MUC1.9 (p < 0.01); Results were obtained from pooled blood samples from 8 to 16 mice in each group. towards MUC1 in MUC1 transgenic mice. IgG2a antiMUC1 was detected only in mice immunized with the combined vaccine $(\mathrm{p}<0.01)$. This finding is in accord with the conclusion based on cytokines profile (Section 3.3.1) that Fla-MUC1.7 and Fla-MUC1.9 induce a Th1 type response against MUC1.

\subsection{Fla-MUC1.7 and Fla-MUC1.9 Do Not Induce Autoimmune Response against Normal MUC1 Expressing Tissue}

A major concern regarding the application of cancer vaccines, based on epitopes of tumor-associated antigens, is the risk of inducing an autoimmune response towards healthy organs. From this particular aspect, MUC1 presents the non-negligible advantage that its normal and malignant forms are distinguished by the immune system [3]. To approach this issue we evaluated whether FlaMUC1.7 and Fla-MUC1.9 induce any autoimmune damage in healthy tissues. To that end, we inspected histologically the presence of cellular infiltrates as well as the architectural structure of tissues for damage or any other sign of autoimmunity in organs expressing MUC1 (lung, kidney and liver) in the MUC1 transgenic mice in response to Fla-MUC1 constructs. As shown in Figure 5(a), naive transgenic mice immunized 4 times with the combination of Fla-MUC1.7 and Fla-MUC1.9 revealed no damage to tissues. In transgenic mice bearing tumors, inflammation was identified in all the studied organs collected from mice administered with the combined vaccines as well as those injected with PBS as control (Figure 5(b)). This indicates that the presence of tumor cells leads to tissue damage, but no autoimmune manifestation could be related to the immunization with Fla-MUC1.7 and Fla-MUC1.9.

\subsection{A Recombinant Flagellin MUC1-Based Vaccine with Extended Population Coverage}

In order to design a recombinant flagellin MUC1-based vaccine that would be effective in a wider human population independently of their HLA haplotypes, a larger portion of the extracellular domain of MUC1, including several epitopes, has to be inserted into the flagellin. By using the SYFPEITHI algorithm, a 25 amino acids sequence GVTSAPDTRPAPGSTAPPAHGVTSA was selected and the corresponding recombinant vaccine Fla-MUC1.25 was constructed. The therapeutic activity of Fla-MUC1.25 on the established tumor was compared to that of the combined Fla-MUC1.7 and Fla-MUC1.9 vaccine. Balb/c mice bearing tumors of average size 0.1 $\mathrm{cm}^{3}$ were immunized with these different preparations or with PBS alone. Thirty eight days post injection, tumors grew on average to a size of more than $0.8 \mathrm{~cm}^{3}$ in the PBS control group, whereas in mice immunized with 

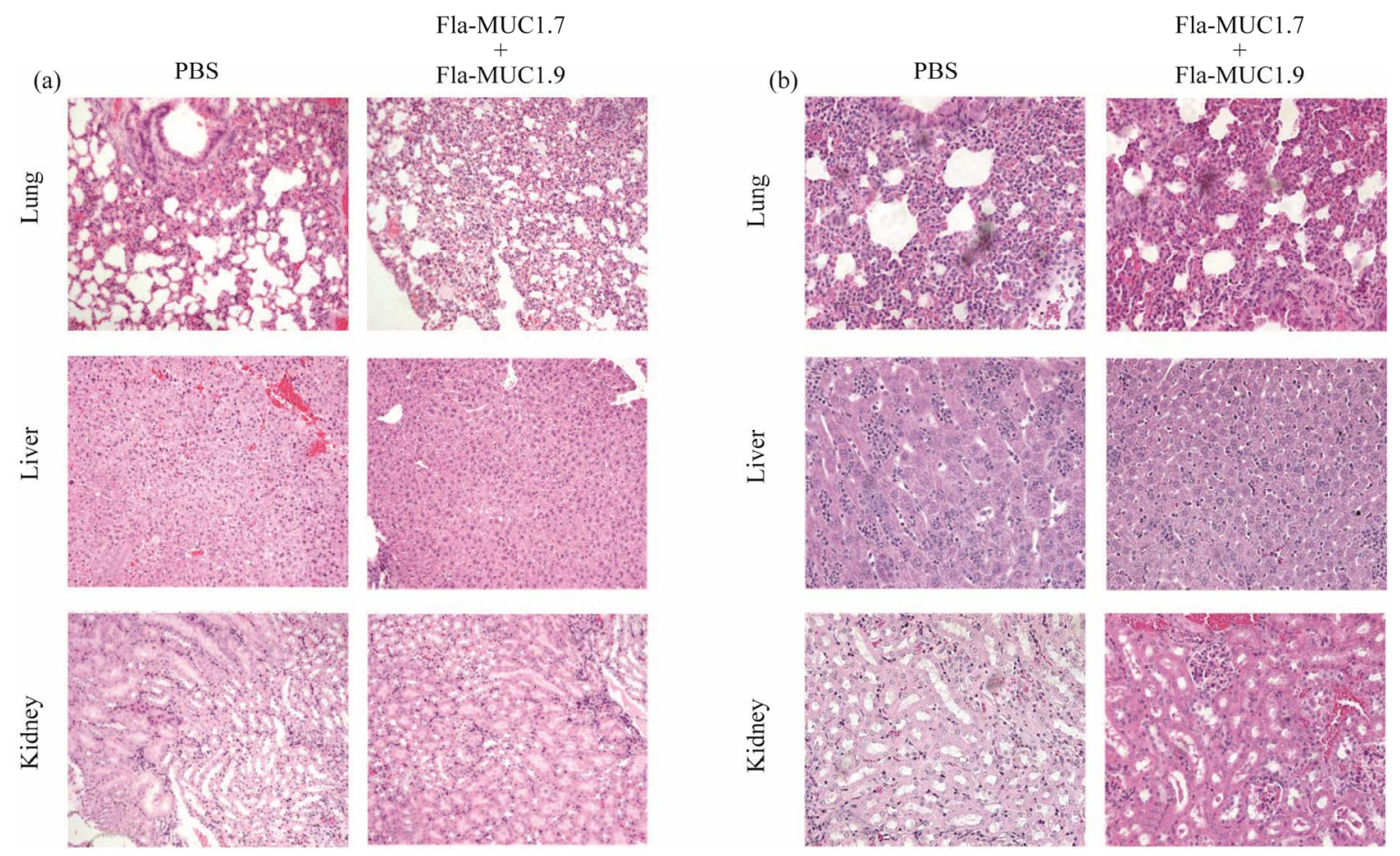

Figure 5. Fla-MUC1.7 and Fla-MUC1.9 do not induce autoimmune response towards healthy tissues. (a) Hematoxylin and eosin staining of lung, liver and kidney from human MUC1 transgenic mice immunized with Fla-MUC1.7 plus Fla-MUC1.9 (denoted FM7 + FM9) or PBS; The bar stands for $100 \mu \mathrm{m}$; (b) Hematoxylin and eosin staining of lung, liver and kidney from human MUC1 transgenic mice bearing 4T1-MUC1 tumors and immunized with Fla-MUC1.7 plus Fla-MUC1.9 (denoted FM7 + FM9) or PBS. The bar stands for $250 \mu \mathrm{m}$.

Fla-MUC1.7 and Fla-MUC1.9 tumor reached the size of $0.4 \mathrm{~cm}^{3}$ (Figure 6(a)). At this same day, in mice immunized with Fla-MUC1.25, the tumors grew in average to less than $0.2 \mathrm{~cm}^{3}$. Furthermore, when these mice were followed for survival determination, $50 \%$ of those FlaMUC1.25 immunized mice survived for over 10 days compared to none in the control (Figure 6(b)). The therapeutic superiority of Fla-MUC1.25 over the mixture composed of Fla-MUC1.7 and Fla-MUC1.9 was confirmed in the transgenic mice (Figure 6(c)). In this same experiment, the number of lung metastases counted individually for each mouse was two-fold lower in both immunized groups as compared to the PBS control group ( $p$ $<0.05$ ) (Figure 6(d)). Furthermore, the size of metastases presented in the lungs of mice vaccinated with Fla-MUC1.25 was clearly smaller in comparison to the lung metastases in the mice immunized with FlaMUC1.7 and Fla-MUC1.9.

\section{Discussion}

Cancer vaccines based on tumor associated-antigens represent an attractive therapeutic strategy as 1) They are aimed at inducing a specific immune response towards the tumor; 2) They are usually not associated with toxic effects; and 3) They can establish a long-term immune memory towards the tumor, which is critical for the pre- vention of tumor recurrence. The tumor associated antigen MUC1 is overexpressed in $90 \%$ of breast cancers and in many other carcinomas such as lung, colorectal, pancreatic, ovarian and prostate cancer. The antigenicity of MUC1 differs between malignant and normal tissue, therefore a MUC1-based cancer vaccine, if effective, could hold a great promise and would be highly valuable in medical oncology.

In this study, we demonstrated the therapeutic activity of several recombinant vaccines expressing different epitopes of MUC1 in flagellin, on both tumor growth and metastases formation. The results with Fla-MUC1.7 are especially interesting considering that this chimeric vaccine carries only a 7 amino-acid MUC1 epitope. The results with the prophylactic immunization show the efficacy of Fla-MUC1.7, after an initial delay of tumor growth in all experimental groups probably due to the use of adjuvant. However, the therapeutic activity of this recombinant MUC1-based vaccine didn't require an external adjuvant. This finding is supported by the already described adjuvant effect of the flagellin and represents a considerable advantage for a potential human application $[8,11,20]$. The SYFPEITHI software was used for the design of Fla-MUC1.9, expressing a 9-amino acid epitope chosen based on its HLA recognition. This construct also demonstrated efficacy in therapeutic vaccination. 
(a)
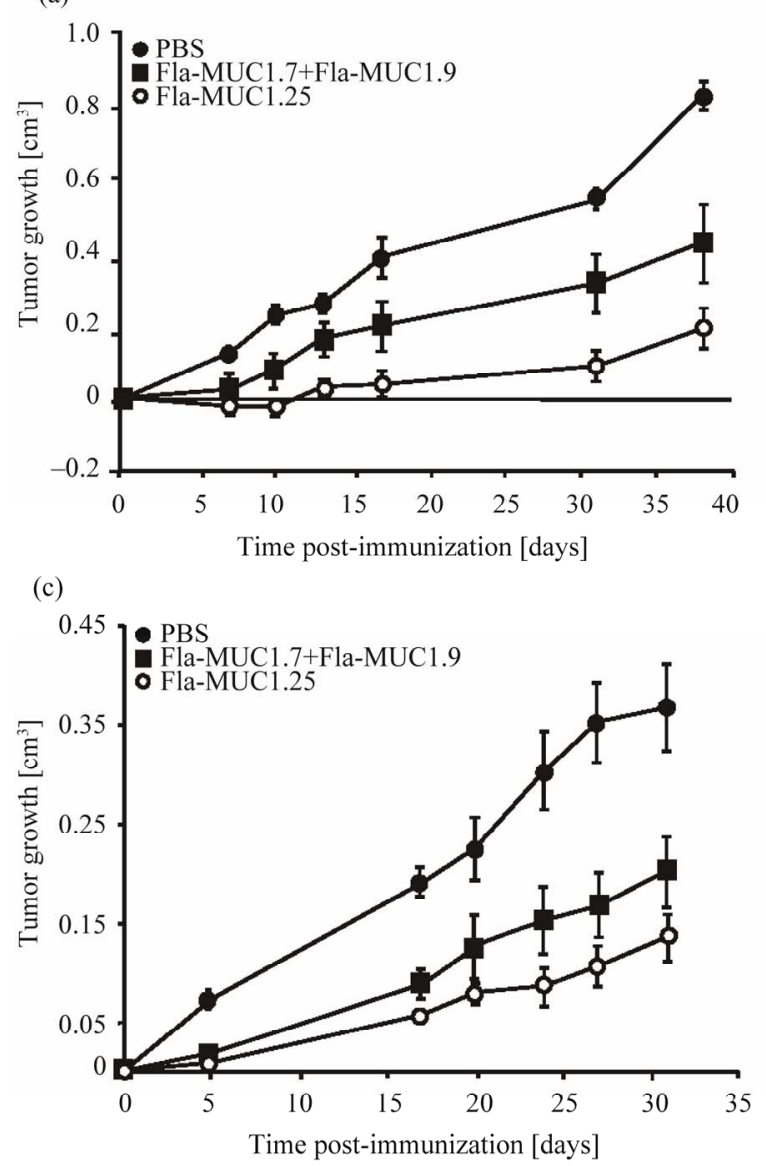

(b)

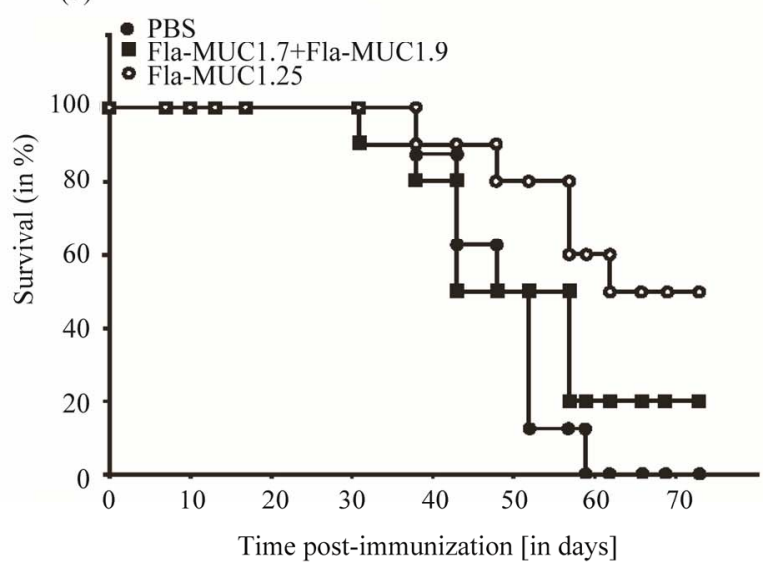

(d)

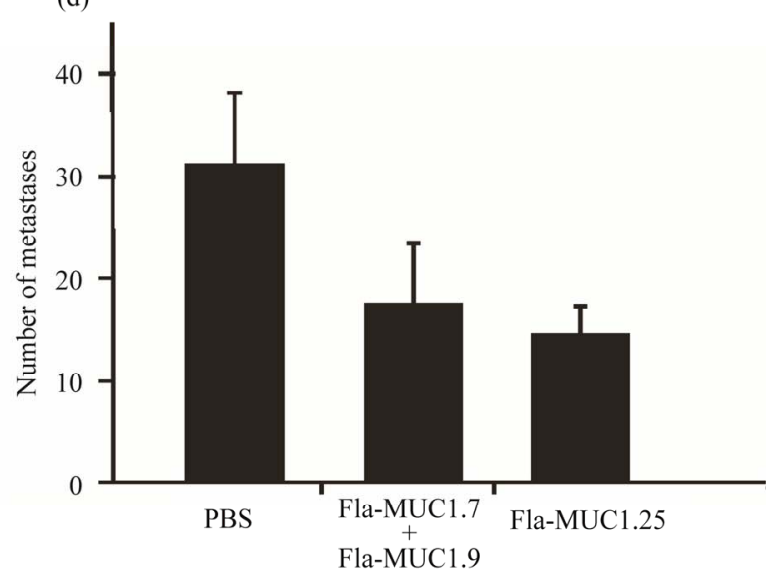

Figure 6. Fla-MUC1.25 reduces tumor growth and metastasis number. Full circle: PBS; Square: Fla-MUC1.7 + Fla-MUC1.9; Empty circle: Fla-MUC1.25. On the day of the immunization (day 0), groups of mice bearing tumor of $0.1 \mathrm{~cm}^{3}$ on the average were immunized with the different preparations as indicated. The average tumor growth (which is the difference between tumor size on a given day and the tumor size on the first day of immunization day 0$) \pm$ s.e. of each group $(\mathrm{n}=10$ to 15$)$ is presented; (a) The reduction of tumor growth observed in Balb/c mice immunized with Fla-MUC1.25 as compared to mice immunized with the mixture Fla-MUC1.7 + Fla-MUC1.9 $(p<0.05)$, in which the tumor growth was reduced as compared to control group $(\mathbf{p}<\mathbf{0 . 0 5})$; (b) Survival of Balb/c mice immunized with Fla-MUC1.25 as compared to control animals; (c) The reduction of tumor growth observed in MUC1 transgenic mice immunized with Fla-MUC1.25 as compared to mice immunized with the mixture Fla-MUC1.7 + Fla-MUC1.9 $(p<0.05)$, in which the tumor growth was reduced as compared to control group (p < 0.05); (d) The number of lung metastases in the control group as compared to mice immunized either with Fla-MUC1.7 + Fla-MUC1.9 or Fla-MUC1.25 $(\mathrm{p}<0.05)$. Lung metastases were visually counted in each mouse on the last day of the experiment described in (c).

Moreover, the combined administration of the two recombinant Flagella MUC1 vaccines, Fla-MUC1.7 and Fla-MUC1.9, led to an additive therapeutic effect. Finally, Fla-MUC1.25, also designed using this database in order to expand the target population to which this new therapeutic approach could be offered, exerted even higher effect on tumor growth, number of lung metastasis and survival.

In some of the experiments, the control construct FlaNRP expressing a non-relevant epitope displayed an anti-tumor activity, though of lower level, in Balb/c mice. This effect might be due to the enhancement, by the flagella, of the existing immune response developed by the $\mathrm{Balb} / \mathrm{c}$ host towards the foreign molecule (MUC1) ex- pressed on the tumor. This is essentially an adjuvant effect of the flagella. Similar observations were reported by another research group [21]. However, the specific therapeutic effect induced by the MUC1 epitopes vaccine was markedly and significantly higher.

The immune response triggered by the flagellin via its ligand the Toll-like receptor 5 is controversial in the literature, and body of data demonstrated the induction of either Th1 or Th2 type response [22-25]. The discrepancy of these observations might be due to the experimental conditions. In our study, we showed that the recombinant flagellin evoked a Th1 response toward the inserted epitope, as evidenced by the INF $\gamma$ secretion by lymphocyte isolated in response to MUC1 stimuli, whereas 
the Th2 response appeared weak.

The use of flagella as a carrier for epitope expression encounters a certain limitation: for its activity to be sustained, the flagellin three-dimensional structure must be conserved [26], which implies that the size of the oligonucleotide that can be inserted into the flagellin gene is confined. Therefore, the design of a recombinant vaccine efficient in a wider human population independently of their HLA haplotypes had to take into consideration the need to carry all the possible epitopes included in a tandem repeat and at the junction of 2 tandem repeats, but also the need to minimize the length of the inserted segment. The production of such recombinant vaccine, which met technical issues while using the current procedure, required a slight modification of the plasmid (described in Section 2.4). Initially, three recombinant vaccines carrying respectively 20,22 and 25 amino acids (which had been chosen using the SYFPEITHI database [17]) were produced. All three constructs led to motile bacteria, indicating that the three-dimensional structure of flagellin was maintained. Hence, the recombinant vaccine carrying the longest MUC1 sequence (namely FlaMUC1.25) was selected for evaluation of the therapeutic vaccination capacity.

One mechanism used by tumor cells to escape the immune system is the down-regulation of expression of MHC class I molecules [27]. Targeting the tandem repeat of the extracellular domain of MUC1 in a cancer vaccine represents a significant advantage since MHC-unrestricted recognition by CTL in humans has been reported in the case of MUC1, probably due to the manifold tandem repeats [28]. Indeed, several clinical trials, examining MUC1 TR -based vaccines, have been conducted in the last few years, and have shown encouraging results in patients with different carcinomas [29-34].

It should be borne in mind that the vaccines described in our study were tested and evinced therapeutic activity in a particularly aggressive animal model. Indeed, the 4T1-MUC1 line is capable to form a tumor upon implantation into Balb/c mice, whereas usually the immunogenicity of MUC1 does not allow MUC1-expressing tumor cells to grow in wild-type mice [16]. This can be attributed to the conservation and expression of the aggressive phenotype of the parental 4T1 line, equivalent to grade IV breast cancer (www.atcc.org), by the 4T1MUC1 line. Furthermore, since the human MUC1 transgenic mice had been shown to be tolerant to MUC1 [16], the 4T1-MUC1 implanted in this particular host, can be considered as a grade IV breast cancer model. This is further supported by the spontaneous metastasis formation of the 4T1-MUC1 line upon implantation and tumor formation in the human MUC1 transgenic mice, as is reported in this study. It should be noted that tumor growth variability inter experiments was observed, but is expected while using such in vivo model. It can be due to many factors including the condition of the cells at implantation time, diffusion of cells under the skin upon injection or the natural immune response occurring in Balb/c to human MUC1 on 4T1-MUC1 expressing cells.

In this study, we investigated the possible advantage of a multiple immunizations schedule, over a single administration of the vaccine. The specific schedule consisting of 3 immunizations at weekly intervals didn't achieve higher therapeutic capacity than the single administration. The design of such study met a non negligible limitation associated with the aggressiveness of the models used in this study. Indeed, the rapid growth of the tumor required euthanasia and thus ending of the experiment within a relatively short time after the initial tumor detection (which occurred 2 weeks after tumor cell implantation). Consequently, a schedule consisting of at least a few weeks intervals between administrations of the vaccine, in order to allow the immune response to go down before the booster immunization, couldn't be explored.

The strategy based on the recombinant flagella MUC1expressing vaccine offers two significant advantages: first, no adjuvant is required in the therapeutic application. This is a highly advantageous feature since new, safer and more efficient adjuvants adequate for human application have yet to be developed [35]. The second advantage is relevant to the use of synthetic peptides in general since they can be highly characterized, are considered safe with no adverse effects and can be synthesized under the good manufacturing practice conditions on an industrial scale [36]. Similarly, the synthetic recombinant flagella constructs are similarly relatively easy to produce. Furthermore, an important clinical observation supports the potential of flagella in cancer vaccine. Indeed, it has been demonstrated that the activation of the Toll-like receptor pathway blocks the suppressive effect of CD4 + CD25 + T regulatory cells on the adaptive immune response [37]. On the other hand, the level of this cell type in peripheral blood of cancer patients is strongly elevated [6]. Hence, this increase could be counteracted by the flagella that are known as ligand of TLR 5 [15].

It is crucial to emphasise that our data demonstrate that recombinant flagella-MUC1-based cancer vaccines are efficient agents against solid growing tumors. It would be reasonable to assume that these vaccines will be efficacious in additional animal models for other MUC1 expressing tumors, such as the lung, liver or pancreas for which the current prognosis is very poor.

\section{Acknowledgements}

We thank Prof. S Gendler (Mayo Clinic Arizona, Scottsdale, USA) for generously offering the human MUC1 
transgenic mice and Prof. Allen T. (University of Alberta, Edmonton, Canada) for kindly giving us the 4T1-MUC1 cell line.

\section{REFERENCES}

[1] O. J. Finn, "Cancer Vaccines: Between the Idea and the Reality," Nature Reviews Immunology, Vol. 3, No. 8, 2003, pp. 630-641. doi:10.1038/nri1150

[2] S. B. Ho, G. A. Niehans, C. Lyftogt, P. S. Yan, D. L. Cherwitz, et al., "Heterogeneity of Mucin Gene Expression in Normal and Neoplastic Tissues," Cancer Research, Vol. 53, No. 3, 1993, pp. 641-651.

[3] J. Taylor-Papadimitirou, J. Burchell, D. W. Miles and M. Dalziel, "MUC1 and Cancer," Biochimica et Biophysica Acta, Vol. 1455, No. 2-3, 1999, pp. 301-313. doi:10.1016/S0925-4439(99)00055-1

[4] M. A. McGuckin, M. D. Walsh, B. G. Hohn, B. G. Ward and R. G. Wright, "Prognostic Significance of MUC1 Epithelial Mucin Expression in Breast Cancer," Human Pathology, Vol. 26, No. 4, 1995, pp. 432-439. doi:10.1016/0046-8177(95)90146-9

[5] A. M. Vlad, J. C. Kettel, N. M. Alajez, C. A. Carlos and O. J. Finn, "MUC1 Immunobiology: From Discovery to Clinical Applications," Advances in Immunology, Vol. 82, 2004, pp. 249-293. doi:10.1016/S0065-2776(04)82006-6

[6] E. Yang, X. F. Hu and P. X. Xing, "Advances of MUC1 as a Target for Breast Cancer Therapy," Histology and Histopathology, Vol. 22, No. 8, 2007, pp. 905-922.

[7] S. M. Newton, C. O. Jacob and B. A. Stocker, "Immune Response to Cholera Toxin Epitope Inserted in Salmonella Flagellin," Science, Vol. 244, No. 4900, 1989, pp. 70-72. doi:10.1126/science. 2468182

[8] R. Levi and R. Arnon, "Synthetic Recombinant Influenza Vaccine Induces Efficient Long-Term Immunity and Cross-Strain Protection," Vaccine, Vol. 4, No. 1, 1996, pp. 85-92. doi:10.1016/0264-410X(95)00088-I

[9] T. Ben-Yedidia, R. Tarrab-Hazdai, D. Schechtman and R. Arnon, "Instranasal Administration of Synthetic Recombinant Peptide-Based Vaccine Protects Mice from Infection by Schistosoma Mansoni," Infection and Immunity, Vol. 67, No. 9, 1999, pp. 4360-4366.

[10] T. Ben-Yedidia, H. Marcus, Y. Reisner and R. Arnon, "Intranasal Administration of Peptide Vaccine Protects Human/Mouse Radiation Chimera from Influenza Infection," International Immunology, Vol. 11, No. 7, 1999, pp. 1043-1051. doi:10.1093/intimm/11.7.1043

[11] A. N. Honko, N. Siranganathan, C. J. Lees and S. B. Mizal, "Flagellin Is an Effective Adjuvant for Immunization against Lethal Respiratory Challenge with Yersinia Pestis," Infection and Immunity, Vol. 74, No. 2, 2006, pp. 1113-1120. doi:10.1128/IAI.74.2.1113-1120.2006

[12] B. L. Whittle, E. Lee, R. C. Weir and N. K. Verma, "Immune Response to a Murray Valley Encephalitis Virus Epitope Expressed in the Flagellin of an Attenuated Strain of Salmonella," Journal of Medical Microbiology, Vol. 46, No. 2, 1997, pp. 129-138. doi:10.1099/00222615-46-2-129
[13] N. K. Verma, H. K. Ziegler, B. A. Stocker and G. K. Schoolnick, "Induction of a Cellular Immune Response to a Defined T-Cell Epitope as an Insert in the Flagellin of a Live Vaccine Strain in Salmonella," Vaccine, Vol. 13, No. 3, 1995, pp. 235-244. doi:10.1016/0264-410X(95)93308-V

[14] C. Cuadros, F. J. Lopez-Hernandez, A. L. Dominguez, M. McClelland and J. Lustgartent, "Flagellin Fusion Proteins as Adjuvants or Vaccines Induce Specific Immune Response," Infection and Immunity, Vol. 72, No. 5, 2004, pp. 2810-2816. doi:10.1128/IAI.72.5.2810-2816.2004

[15] D. Van Duin, R. Medzhitov and A. C. Shaw, "Triggering TLR Signalling in Vaccination," Trends in Immunology, Vol. 27, No. 1, 2006, pp. 49-55. doi:10.1016/j.it.2005.11.005

[16] T. Ben-Yedidia and R. Arnon, "Effect of Pre-Existing Carrier Immunity on the Efficacy of Synthetic Influenza Vaccine," Immunology Letters, Vol. 64, No. 1, 1998, pp. 9-15. doi:10.1016/S0165-2478(98)00073-X

[17] G. H. Rammensee, J. Bachmann, N. N. Emmerich, O. A. Bachor and S. Stevanovic, "SYFPEITHI: Database for MHC Ligands and Peptide Motifs," Immunogenetics, Vol. 50, No. 3-4, 1999, pp. 213-219. www.syfpeithi.de doi:10.1007/s002510050595

[18] E. H. Moase, W. Qi, T. Ishida, Z. Gabos, B. M. Longenecker, et al., "Anti-MUC-1 Immunoliposomal Doxorubicin in the Treatment of Murine Models of Metastatic Breast Cancer," Biochimica et Biophysica Acta, Vol. 1510, No. 1-2, 2001, pp. 43-55. doi:10.1016/S0005-2736(00)00334-5

[19] G. J. Rowse, R. M. Tempero, M. L. VanLith, M. A. Hollingworth and S. J. Gendler, "Tolerance and Immunity to MUC1 in a Human MUC1 Transgenic Murine Model," Cancer Research, Vol. 58, No. 2, 1998, pp. 314-321.

[20] S. J. McSorley, B. D. Ehst, Y. Yu and A. T. Gewirtz, "Bacterial Flagellin is an Effective Adjuvant for CD4+ T Cells in Vivo," The Journal of Immunology, Vol. 169, No. 7, 2002, pp. 3914-3919.

[21] L. Sfrondini, A. Rossini, D. Besusso, A. Merlo, E. Tagliabue, et al., "Antitumor Activity of the TLR-5 Ligand Flagellin in Mouse Models of Cancer," The Journal of Immunology, Vol. 176, No. 11, 2006, pp. 6624-6630.

[22] L. Strindelius, M. Filler and I. Sjoholm, "Mucosal Immunization with Purified Flagellin from Salmonella Induces Systemic and Mucosal Immune Response in CH3/HEJ Mice," Vaccine, Vol. 22, No. 27-28, 2004, pp. 3797-3808. doi:10.1016/j.vaccine.2003.12.035

[23] S. Agrawal, A. Agrawal, B. Doughty, A. Gerwitz, J. Blenis, et al., "Cutting Edge: Different Toll-Like Receptor Agonists Instruct Dendritic Cells to Induce Distinct Th Responses via Differential Modulation of Extracellular Signal-Regulated Kinase-Mitogen-Activated Protein Kinase and c-Fos," The Journal of Immunology, Vol. 171, No. 10, 2003, pp. 4984-4989.

[24] A. Didierlaurent, I. Ferrero, L. A. Otten, B. Dubois, M. Reinhardt, et al., "Flagellin Promotes Myeloid Differentiation Factor 88-Dependent Development of Th2-Type Response," The Journal of Immunology, Vol. 172, No. 11, 2004, pp. 6922-6930. 
[25] R. M. Salazar-Gonzalez and S. J. McSorley, "Salmonella Flagellin a Microbial Target of the Innate and Adaptive Immune System," Immunology Letters, Vol. 101, No. 2, 2005, pp. 117-122. doi:10.1016/j.imlet.2005.05.004

[26] S. Ho Jeon, T. Ben-Yedidia and R. Arnon, "Intranasal Immunization with Synthetic Recombinant Vaccine Containing Multiple Epitopes of Influenza Virus," Vaccine, Vol. 20, No. 21-22, 2002, pp. 2772-2780. doi:10.1016/S0264-410X(02)00187-1

[27] P. Zheng, S. Sarma, Y. Guo and Y. Liu, "Two Mechanisms for Tumor Evasion of Preexisting Cytotoxic T-Cell Responses: Lessons from Recurrent Tumors," Cancer Research, Vol. 59, No. 14, 1999, pp. 3461-3467.

[28] N. M. Alajez, J. Schmielau, M. D. Alter, M. Casio and O. J. Finn, "Therapeutic Potential of a Tumor-Specific MHC-Unrestricted T-Cell Receptor Expressed on Effector Cells of the Innate and the Adaptive Immune System through Bone Marrow Transduction and Immune Reconstitution," Blood, Vol. 105, No. 12, 2005, pp. 4583-4589. doi:10.1182/blood-2004-10-3848

[29] R. K. Ramanathan, K. M. Lee, J. McKolanis, E. Hitbold, W. Schraut, et al., "Phase I Study of a MUC1 Vaccine Composed of Different Doses of MUC1 Peptide with SBAS2 Adjuvant in Resected and Locally Advanced Pancreatic Cancer," Cancer Immunology, Immunotherapy, Vol. 54, No. 3, 2004, pp. 254-264. doi:10.1007/s00262-004-0581-1

[30] B. Acres, "Cancer Immunotherapy: Plase II Clinical Studies with TG4010 (MVA-MUC1-IL2)," Journal of B.U.ON.: Official Journal of the Balkan Union of Oncology, Vol. 12, Suppl. 1, 2007, pp. S71-S75.

[31] V. Apostolopoulos, G. A. Pietersz, A. Tsibanis, A. Tsikkinis, H. Drakaki, et al., "Pilot Phase III Immunotherapy Study in Early Stage Breast Cancer Patients Using Oxidized Mannan-MUC1," Breast Cancer Research, Vol. 8, No. 3, 2006, pp. 27-32. doi:10.1186/bcr1505

[32] R. Sangha and S. North, "L-BLP25: A MUC1 Targeted Peptide Vaccine Therapy in Prostate Cancer," Expert Opinion on Biological Therapy, Vol. 7, No. 11, 2007, pp. 1723-1730. doi:10.1517/14712598.7.11.1723

[33] J. Wierecky, M. R. Müller, S. Wirths, E. Halder-Oehler, D. Dörfel, et al., "Immunologic and Clinical Responses after Vaccinations with Peptide-Pulsed Dendritic Cells in Metastasic Renal Cancer Patients," Cancer Research, Vol. 66, No. 11, 2006, pp. 5910-5918. doi:10.1158/0008-5472.CAN-05-3905

[34] K. Yamamoto, T. Ueno, T. Kawaoka, S. Hazama, M. Fukui, et al. "MUC1 Peptide Vaccination in Patients with Advanced Pancreas or Biliary Tract Cancer," Anticancer Research, Vol. 25, No. 5, 2005, pp. 3575-3579.

[35] D. T. O'Hagan, M. L. MacKichan and M. Singh, "Recent Developments in Adjuvants for Vaccines against Infectious Diseases," Biomolecular Engineering, Vol. 18, No. 3, 2001, pp. 69-85. doi:10.1016/S1389-0344(01)00101-0

[36] N. Yajima, R. Yamanaka, T. Mine, N. Tsuchiya, J. Homma, et al., "Immunologic Evaluation of Personalized Peptide Vaccination for Patients with Advanced Malignant Glioma," Clinical Cancer Research, Vol. 11, No. 16, 2005, pp. 5900-5911. doi:10.1158/1078-0432.CCR-05-0559

[37] C. Pasare and R. Medzhitov, "Toll Pathway-Dependent Blockade of CD4+CD25+ T Cell-Mediated Suppression by Dendritic Cells," Science, Vol. 299, No. 5609, 2005, pp. 1033-1036. doi:10.1126/science. 1078231 